\title{
STUDIES OF SNRS IN THE MAGELLANIC CLOUDS
}

\author{
A.J. TURTLE \\ School of Physics \\ University of Sydney \\ Sydney NSW 2006 \\ Australia
}

ABSTRACT. In the Magellanic Clouds about 60 confirmed supernova remnants (SNRs) or candidates are known. They have been detected by a combination of X-ray, optical and radio observations. Various statistical aspects of these SNRs will be considered including the implied supernova rate, the spatial distribution, the implications of the radio luminosity/diameter distribution for SNR evolution, and the relation to the extended non-thermal emission. 